\title{
Therapeutic applications of the mobile phone
}

\author{
Alessandra Preziosa ${ }^{\mathrm{a} *}$, Alessandra Grassi ${ }^{\mathrm{b}}$, Andrea Gaggiolia ${ }^{\mathrm{a}, \mathrm{b}}$ and \\ Giuseppe Riva ${ }^{\mathrm{a}, \mathrm{b}}$ \\ ${ }^{a}$ ATN-P Lab, Applied Technology for Neuro-Psychology, Istituto Auxologico Italiano, Milan, \\ Italy; ${ }^{b}$ Department of Psychology, Catholic University of Milan, Italy
}

(Received 12 September 2008; final form received 24 February 2009)

\begin{abstract}
As the availability of new communication technologies increases, mental health professionals have incorporated these innovations into their practice and research. Up to now several studies have presented promising results in using the power and convenience of the Internet for clinical care. While multiple contributions focus on the potential and the advantages of therapies delivered through the Internet, mental health practitioners may take into account new technological opportunities to improve their practice. Mobile phone diffusion is expanding worldwide at breath-taking speed. In fewer than 20 years, mobile phones have gone from being rare and expensive pieces of equipment used primarily by the business elite, to a pervasive low-cost personal item. In many countries, mobile phones now outnumber land-line telephones, with most adults and many children now owning mobile phones. With high levels of mobile telephone penetration, a mobile culture has evolved, where the phone becomes a key social and cultural tool. The purpose of this paper is to explore the potentialities that mobile phones may offer in clinical care. The paper will investigate in which areas of clinical interventions mobile phones have already been successfully applied. Moreover, the paper will discuss these opportunities by presenting the results of two different studies based on the use of the mobile phone for anxiety management.
\end{abstract}

Keywords: mobile phone; psychotherapy; assessment; counselling

\section{Background}

During recent years the advance of technology offers new communication tools that mental health professionals and patients feel comfortable using for clinical care. As the availability of new communication technologies and services, like the Internet, expands the ways in which treatment can be provided, professionals will incorporate these innovations into their practice and research. From this idea, recently there has been a growth of e-therapy: the use of the Internet and related media for clinical care. Some pioneering works have presented promising results in Internet-aided psychotherapy applied for both individual therapy and self-help therapy. For example, an individual therapy intervention for patients with panic disorder provided by the Internet was investigated by Klein and Richards (2001). In their work the Internet-based treatment revealed significant reductions in measures pertaining to panic, negative affect, body vigilance, self-efficacy and managing panic. Moreover Jordan (2005) presented an Internet-based information and counselling project for the prevention of drug addiction

*Corresponding author. Email: a.preziosa@auxologico.it 
(www.drugcom.de). Another pioneering method of individual online therapeutic intervention for gamblers was developed by the Gordon House Association and Datasmith Ltd. (www.gamblingtherapy.org). This online software came about as a method of post-residential support and outreach for clients (Anthony, 2005).

Self-help materials that patients have widely at their disposal on the web may be selfadministered with or without the therapist's guidance. The Internet enables self-help procedures for a wide variety of psychological problems such as phobias, obesity, sexual dysfunctions and tobacco addiction. According to a meta-analysis, that reviewed 40 well-designed outcome studies of online self-help treatment, online self-help appears to be more effective than no treatment at all and just as effective in most cases as treatment administered by the therapist (Scogin, Bynum, Stevens, \& Calhoon, 1990). Madara (1990) demonstrates that online self-help groups improve social support, practical information, shared experiences, positive role models, helper therapy, empowerment, professional support and advocacy efforts, just as face-to-face groups do.

This overview suggests that Internet and communication media could lead to possible significant mental health initiatives. Multiple contributions focus on the potential and the advantages (Budman, 2000; Griffiths, 2001), the dangers and disadvantages (Anthony, 2000; Baur, 2000; Griffiths, 2001), and the legal and ethical aspects of therapies delivered through the Internet (Bloom, 1998; Maheu \& Gordon, 2000; Manhal-Baugus, 2001) and there is wide agreement in considering this communication technology strictly linked to home usage. Individuals have to be very interested and motivated in its use for their clinical treatment. Moreover, etherapy needs technological requirements that are not so widely diffused.

On the other hand, nowadays such views ignore the basic facts that in comparison to PC and Net technologies, other communication technologies are used by broader strata of the population all over the world. In particular, mobile phones have stronger impacts on social life, so that most people are ready to spend much larger sums of money on monthly phone bills than on Internet provider services.

Over the last few years, mobile phone density has increased rapidly. While mobile phone penetration in Europe was $70 \%$ by the end of the 2001, it increased to $85 \%$ in 2005 and today it totals around 34 million subscriptions (Table 1). With this high level of mobile phone penetration, a 'mobile culture' has evolved, where the phone

Table 1. (a) Total number of mobile phone subscriptions in Europe (in millions); (b) Mobile phone penetration in Europe, 2000-2005.

(a)

\begin{tabular}{lrrrc}
\hline Region & $\mathbf{2 0 0 2}$ & $\mathbf{2 0 0 3}$ & $\mathbf{2 0 0 4}$ & $\mathbf{2 0 0 5}$ \\
\hline Northern Europe & 24 & 25 & 25 & 25 \\
Southern Europe & 70 & 75 & 78 & 80 \\
Central W. Europe & 160 & 170 & 175 & 178 \\
\hline
\end{tabular}

(b)

\begin{tabular}{lc}
\hline Year & Total $\%$ \\
\hline $\mathbf{2 0 0 0 - 1}$ & $70 \%$ \\
$\mathbf{2 0 0 5}$ & $85 \%$
\end{tabular}

Source: Statistics for mobile commerce on line (http:II www.epaynews.com). 
becomes a key social and cultural tool. Thus, mobile phones have become popular even in rather 'technophobic contexts like Italy, where computers and other modern technologies have a difficult stand' (Fortunati, 2002) and in Scandinavian countries where people traditionally are introverted and silence in talk is highly valued (Puro, 2002). For adolescents, observations and teenagers' own accounts have shown that the ubiquity of mobile phone use is, in general, commonplace and phone-mediated activities are a routine, taken-for-granted part of teenagers' daily encounters (Taylor \& Harper, 2001).

A recent empirical study by the International Telecommunications Union provides striking evidence of how the mobile phone has contributed to narrowing the century-old gap in telephone usage between highly developed and less developed countries. It shows that in 2001 about 100 nations (among them many African countries) had more mobile than landline phones in service and that mobile phone technology is far more potent than computer technology in connecting less privileged populations to the sphere of digitalised information (World Telecommunication Development Report, 2002).

There is wide agreement that hand-held phone sets can substitute stationary PCs or mobile laptops to a considerable degree because they are in the course of becoming multimedia devices able to transport voice, text messages, pictures, musical sounds, software programs and anything else coded in digital format. A new generation of $3 \mathrm{G} / \mathrm{UMTS}$ and $4 \mathrm{G}$ wireless terminals, in fact, will also provide advanced 3D graphics and broader communication capabilities. In addition, these multimedia functionalities are combined with significantly reduced size, weight, energy needs and purchase price, as well as by a much simpler, more user-friendly interface, which makes it possible for them to be used by younger people, disabled people and other marginal population segments.

Nevertheless, the mobile phone seems to evoke much less intellectual enthusiasm and scientific research endeavours than the World Wide Web. For instance, only the Internet is given the status of a Mega-Innovation that really counts, while mobile communication facilities are almost totally neglected (Castells, 1996).

Starting from these specific mobile phone capabilities, several considerations demonstrate that the use of this tool may respond to several clinical needs. First of all, clinical practice is especially based on the patient-therapist relationship: this bond does not collapse when patients leave the therapist's consulting room, but has to continue in daily life. Another critical issue is compliance with the treatment which improves the effectiveness of the care. Finally, key factors are resilience and coping skills: incrementally, the acquisition of these abilities in a therapeutic setting make the patient able to cope with everyday situations in an autonomous way (Storr, 1990). Drawing attention to these particular clinical needs, the critical benefits that mobile phones may introduce in clinical practice are:

- Mobile phones are one of the most widespread technologies. The wide diffusion of this technology reduces the problems of the digital divide and offers individuals of various groups the possibility of research and treatment access.

- Advanced multimedia capabilities and speed in connection facilitate interactive feedback, increasing participants' compliance to the treatment. Moreover, this process improves the transfer of the abilities acquired in a therapeutic setting to everyday life. 
- Mobile phones guarantee the availability of the contents any time and everywhere: in this sense their portability would be an eligible feature for assessments done in the patient's context.

- The common use in life of mobile phones may enhance patients' acceptance of its application in mental health education and treatment.

- High connection speeds may offer new opportunities for a quick transfer and management of data for the therapist's practice.

To explore these opportunities, in the next section some pioneering studies in mobile phone application for clinical care will be presented. More in detail, the aim of the presentation is to investigate in which areas of clinical interventions mobile phones have already been successfully applied. In conclusion, new suggestions for the use of the mobile phone in clinical practice will be introduced.

\section{Applications of the mobile phone in clinical care}

Mobile phone usage has already been harnessed in health care generally, but in the last few years this application is being explored also in the mental health field.

In the health care area, several researchers have been involved in defining the role of mobile devices, such as the mobile phone, to record health information about patients. Researchers have demonstrated that linking mobile phone technology with electronic data collection supports the recording of many patients' information about their status and improves their compliance with the treatment (Braun et al., 2005; Cleland, Caldow, \& Ryan, 2007). The use of the mobile phone in clinical practice facilitates communication with a health care professional in the case where face-toface consultation is impossible. Moreover, there has been a high degree of acceptability for both patients and staff (Cleland et al., 2007). Studies have been conducted to determine the effectiveness of one of the functions of the mobile phone, such as text messaging, in improving the attendance rate in primary care (Leong et al., 2006), in reminding about drug intake and in forwarding educational messages to patients with chronic diseases (Neville, Greene, McLeod, \& Tracy, 2002). Results suggest that text messages that are reminders about treatment and useful tips on education may be a medium to allow people with chronic health problems to make their disease comply with their lifestyle and not the other way around (Neville et al., 2002).

These findings in the general health area have also led to the decision to choose text messaging on mobile phones also for use in the aftercare of patients with mental illness. A pilot study carried out at a German hospital indicates how text messaging on mobile phones was used in the treatment of bulimia. The intervention, offered for six months following discharge from inpatient psychotherapy, consisted of weekly messages from the patients on their bulimic symptomatology and a corresponding weekly feedback. The study was designed to test the acceptance, the practicability and the effectiveness of the mobile device intervention. Preliminary results indicate that the programme was well accepted and gave support to bulimic patients after finishing inpatient treatment (Bauer, Percevic, Okon, Meermann, \& Kordy, 2003). This study was repeated in the UK (Robinson et al., 2006). In this case the participants' low response rate and answers to the questionnaire suggest that the intervention was only moderately well accepted by participants. However, at least a subgroup of participants felt supported and encouraged by the feedback messages. 
The number of outpatient sessions that participants had attended varied greatly, with some having only received brief treatment before starting the programme. This finding contrasts with the German study where patients had all undergone a lengthy in-patient programme and may therefore have been more prepared to invest time in staying well. In a current study Joyce \& Weibelzahl (2006) are investigating how text messaging on mobile phones can be harnessed to encourage students who suffer from depression to overcome barriers of help seeking, by initiating dialogue and providing timely exhortations to avail themselves of counselling services.

Two more studies have indicated that text messaging can be used to help college students successfully quit smoking. Obermayer, Riley, Asif, and Jean-Mary (2004) developed and evaluated a prototype programme, targeting college students of the United States, that integrates Web and mobile phone technologies to deliver a smoking cessation intervention. Forty-six regular smokers were recruited from local colleges and provided with access to the programme. At the six-week follow-up, $43 \%$ had made at least one 24 -hour attempt to quit, and $22 \%$ had quit, based on a sevenday prevalence criterion. Rodgers et al. (2005) recruited 1705 smokers from throughout New Zealand who wanted to quit, were aged over 15 years, and owned a mobile phone. Participants were allocated at random to an intervention group that received regular, personalised text messages providing smoking cessation advice, support and distraction, or to a control group. Results showed that more participants had quit at six weeks into the intervention compared to the control group.

Although encouraging, these pioneering studies have confirmed that only a little of the enormous potential of mobile technology has already been explored. These studies investigated the effectiveness of the text messaging feature in clinical care. In particular they were involved in defining the key role played by the ubiquity of mobile phones in anchoring the patient to the clinical treatment, anywhere and any time. In any case, the contribution of other mobile phone capabilities for clinical care is neglected. As defined before, extensive mobile phone handsets and evolved services, such as videoconferencing and video streaming, are being developed. And the contribution offered by multimedia contents on the mobile phone has still not been investigated. Starting from these focal points, new and exploratory mobile phone contributions are suggested on two levels:

\section{Assessment and follow-up}

It is possible to assess/improve the outcome of the clinical treatment through the mobile phone. The studies referring to the use of text messaging on the mobile phone have confirmed that the continuous monitoring of patient treatment response with immediate feedback to therapists can be used to increase the likelihood of success for the poorly responding client (Bauer et al., 2003; Joyce \& Weibelzahl, 2006; Obermayer et al., 2004; Rodgers et al., 2005). A current study proposes to test the use of the mobile phone for assessing everyday experiences.

This work presents the implementation of an ecological assessment methodology on mobile phones for reaching and monitoring patients' information related to their experience in natural settings (Preziosa, Bassi, Villani, Gaggioli, \& Riva, 2006). Participants are asked to fill out a questionnaire on their mobile phone when it emits acoustic signals, reporting on their current activities, location, social context and associated subjective experience, in terms of motivational, affective and cognitive components. All respondents are tested in two sessions: one with a version of the 
ecological assessment based on paper and pencil, and one with a version implemented on mobile phones. Preliminary results suggest that the use of the mobile phone in filling out the questionnaire shows a higher level of participants' compliance as compared to the traditional paper and pencil version. Other studies have confirmed the feasibility of using the mobile phone in collecting ecological assessment data. This method has useful advantages, including the instantaneous entry of data into a central database, date and time stamping of data and easy integration into daily life. These preliminary studies suggest that the use of mobile phones for assessment is a promising alternative to traditional self-monitoring procedures (Barrett \& Feldman Barrett, 2001; Chen, 2006; Collins, Kashdan, \& Gollnisch 2003; Green, Rafaeli, Bolger, Shrout, \& Reis, 2006). In this case participants feel practised in using a well-known instrument for them, such as the mobile phone, also addressed to other features like psychological assessments.

\section{Counselling and training}

Thanks to the advanced graphic and communication capabilities now available, mobile phones can be used as training/simulation devices to facilitate the transfer of the knowledge acquired in the therapeutic setting to the real life context. To our knowledge, only some ongoing projects are currently experimenting with the role played by mobile phones for acquiring coping skills.

In order to clearly explain the framework that we have discussed so far, the next sections will present two preliminary studies based on the use of mobile phone for anxiety management.

\section{Study 1: The use of mobile phones for managing exam stress}

Exam-taking anxiety or stress is very common among university students. It can be very distressing and sometimes debilitating. Exam anxiety involves physical components and emotional components that may be taken into account for managing and reducing anxiety.

A common approach to control exam anxiety is Stress Inoculation Training (SIT). SIT (Hains, 1992; Saunders, Driskell, Johnston, \& Salas, 1996) has been employed on a treatment basis to help individuals cope with the aftermath of exposure to stressful events and on a preventive basis to 'inoculate' individuals against future and ongoing stressors.

Typically a SIT protocol is based on a three-phase intervention (Coburn \& Manderino, 1986; Meichenbaum, 1996):

- conceptualisation phase: a Socratic-type exchange is used to educate clients about the nature and impact of stress;

- skills acquisition and rehearsal: the specific coping skills are taught to the clients;

- application and follow through: provides opportunities for the clients to apply the variety of coping skills across increasing levels of stressors.

To help students in managing exam stress we developed a specific protocol based on mobile narratives - multimedia narratives experienced on UMTS/3G phones (Nokia 6680). Following the SIT protocol, ours is composed of six sessions: 
- Sessions 1 and 2 target the psycho-physiological reactions to the exam.

- Sessions 3 and 4 target the psycho-physiological reactions and coping strategies.

- Sessions 5 and 6 present the stressful situation (an exam) to test and eventually tune the learned strategies (Figure 1).

According to this framework 30 female university students $(\mathrm{M}=23.48$; $\mathrm{sd}=1.24)$ who were going to take an exam within a week were included in a technology-based treatment for stress management. They were randomly divided into five groups according to the type and mobility of the medium used: (1) audio-only narrative (CD at home); (2) audio-only narrative (portable MP3); (3) audio and video narrative (DVD at home); (4) audio and video narrative (UMTS phone based); (5) control group.

At the start of the treatment, before and after the exam, the subjects were submitted to the following questionnaires: STAI: State-Trait Anxiety Inventory (Spielberger, Gorsuch, Lushene, Vagg, \& Jacobs, 1983); VAS: Visual Analog Scale (Gift, 1989) and PANAS: Positive and Negative Affect Schedule (Watson, Clark, \& Tellegen, 1988).

Results showed that audio/video narratives induced a reduction in exam anxiety in more than $80 \%$ of the sample vs $50 \%$ of the MP3 sample and $0 \%$ of the CD sample (STAI Questionnaire $-X^{2}=11.25 ; p<.05-$ Figure 2). Furthermore, all the users who experienced mobile narratives on UMTS phones were able to relax before the exam, against $50 \%$ of DVD users and $33 \%$ of audio-only users (VAS Questionnaire $-X^{2}=$ 13.33, p $<.05$ - Figure 3).

Finally, a negative correlation was found between the level of anxiety - as assessed by the VAS Questionnaire and exam marks $(\mathrm{r}:-.340, \mathrm{p}<.05)$.

\section{Visual Analogue Scale Questionnaire}

The trial showed a better efficacy of mobile narratives experienced on UMTS phones in reducing the level of exam stress and in helping the student to relax. These results suggest that for the specific sample considered - Italian university students - the media used for providing an anti-stress protocol has a clear impact on its efficacy.

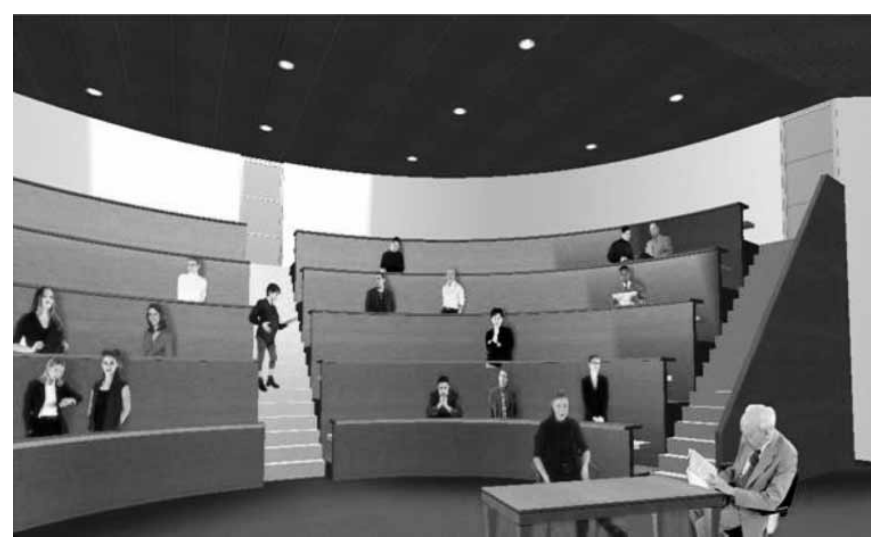

Figure 1. A screen shot of the simulated exam. 


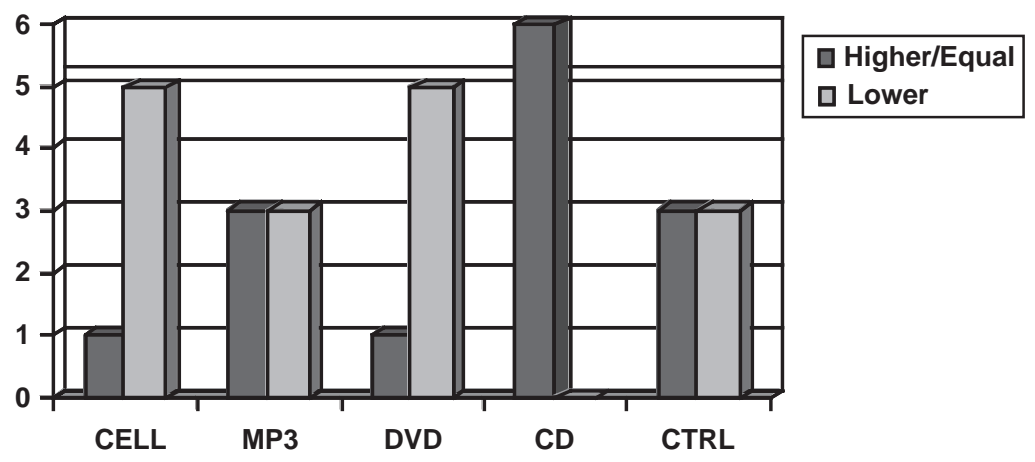

Figure 2. Anxiety level during exam.

Furthermore, the data confirm that $3 \mathrm{G}$ mobile handsets may be used as relaxation tools when backed up by a specific therapeutic protocol and meaningful narratives.

\section{Study 2: Stress management using UMTS mobile phones}

One of the best strategies for dealing with stress is learning how to relax. However, relaxing is difficult to achieve in typical real world situations. In this study, supported by the Italian MIUR FIRB research project, we developed a specific protocol based on mobile narratives, to be experienced on $3 \mathrm{G}$ smart handsets by commuters. Mobile narratives are audio-visual experiences, implemented on mobile devices, in which the narrative component is a critical aspect to induce a feeling of presence and engagement. Through the link between the feeling of presence and the emotional state, mobile narratives may be used to improve the mood state in their users.

The experimental sample included 45 male and 45 female commuters $(\mathrm{N}=90)$ of the Italian regional train line 'Milano-Saronno', aged between 20 and 25 years $(\mathrm{M}=$ $23.48 \pm 1.23)$. The sample was randomly divided between the following three conditions:

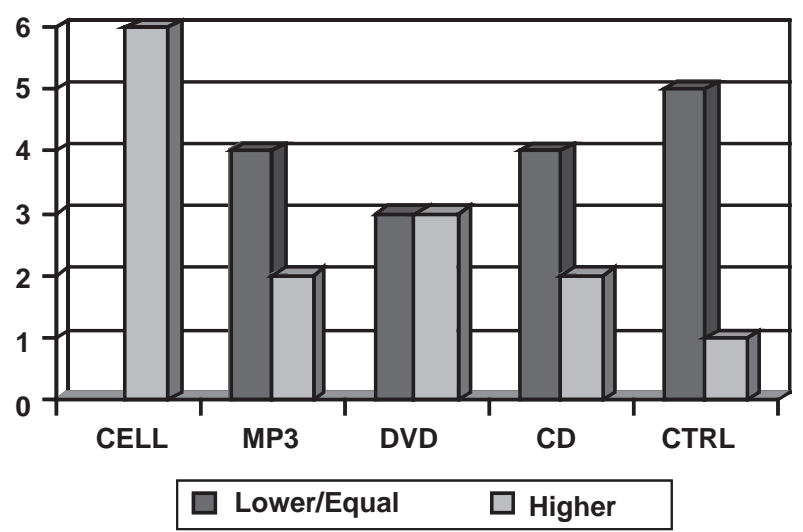

Figure 3. Relaxation level before exam. 
- $\mathrm{MN}$ - Mobile narratives: the sample experienced four mobile narratives based on a trip to a deserted tropical beach (see Figure 4);

- NA - New age videos: the sample experienced four commercial videos with new age music (see Figure 4). The videos were selected for their similar visual content (a tropical beach) to the mobile narratives;

- CT - Control group: no treatment.

The sample was tracked for two days. During each trip the experimental samples experienced the multimedia content on a Motorola A925 UMTS cellular phone (screen size: $208 \times 320$ pixels). The total length of each experience was six minutes. Before and after each trip the subjects were submitted to the following questionnaires: STAI: State-Trait Anxiety Inventory; VAS: Visual Analogue Scale; PANAS: Positive and Negative Affect Schedule; ITC-Sopi: Sense of Presence Inventory.

The first significant result was the difference in anxiety between the three groups. Only the MN group experienced a significant reduction in the anxiety level (STAI: $\left.\mathrm{F}=7,776_{(1.58)}, \mathrm{p}<0.01\right)$ and an increase in the relaxation scale (VAS: $\mathrm{F}=18.993_{(2.87)}$; $\mathrm{p}<0.01$ ) at the end of the trial (Table 2).

The second relevant result is related to the level of presence experienced by the two experimental groups. The level of 'engagement' and 'spatial presence' was significantly higher in the MN group. These data suggest that the efficacy of the MN may be related to the higher level of presence induced by mobile narratives.

The trials showed the efficacy of mobile narratives in reducing the level of stress experienced during a commuting trip. No effects were found in the other groups. These results suggest that $3 \mathrm{G}$ mobile handsets may be used as a relaxation tool if backed up by a specific therapeutic protocol and an engaging experience.

\section{Conclusions}

Nowadays, the majority of programmes have explored the power and convenience of the Internet for the treatment of mental health disorder. This perspective has encountered positive reactions by professionals and patients. However, such views ignore the fact that in comparison with PCs and Net technologies, mobile phones are used today by broader strata of the population all over the world and that, for many

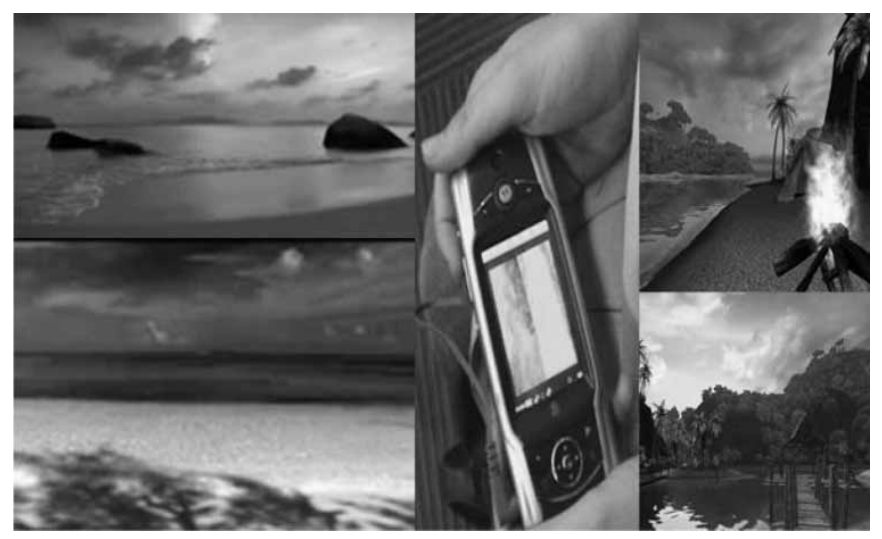

Figure 4. The visual content of the Mobile Narratives (right) and the New Age video (left) 
Table 2. M and Sd: difference from the beginning to the end of the treatment of the anxiety level (assessed by Stai questionnaire) and the relaxation level (assessed by Vas questionnaire).

\begin{tabular}{lcccccc}
\hline & Anxiety & & & \multicolumn{3}{c}{ Relax } \\
\cline { 1 - 3 } \cline { 6 - 7 } Condition & M & Sd & & Condition & M & Sd \\
\hline MN & -3.73 & 5.40 & & MN & 1.37 & 1.10 \\
NA & 0.17 & 5.44 & & NA & -.43 & 1.33 \\
CT & -1.78 & 5.72 & & CT & -.13 & .82 \\
\hline
\end{tabular}

users, they have stronger impacts on social life. Due to this new market-trend, the focus on the potential and the advantages of therapies delivered through technology tools may switch its attention from the Internet to the mobile phone's features. Up to now, taken together, the results of the pioneering studies on the application of mobile phones in clinical practice suggest that this technology is well suited for the purpose: it offers an attractive possibility to bridge the gap between inpatient and outpatient treatment (Bauer et al., 2003).

Currently the mobile phone supports advanced communicational features such as real time video communications, audio, and the exchange of texts and videos. This innovation will increase in the next few years, so a new generation of hardwareaccelerated mobile devices will soon be joined by a suite of emerging 3D software standards that give developers the ability to create interactive content and other applications that have not been possible before.

Starting from the suggestions of the studies that investigate the opportunities offered by communication technologies in clinical care, we have tried to integrate a new perspective for mobile phone use in clinical treatment. The approach proposed in this paper investigated the potentialities of the mobile phone multimedia capabilities for clinical care. In contrast to previous research, the patient can be offered clinical content available any time and anywhere. Patients autonomously selfadminister clinical content, becoming able to improve their knowledge. Using the mobile phone in this way allows them to experience clinical contents, situated not only in a therapeutic setting, but also in an everyday context.

However, the real effectiveness of this tool depends largely on the ability of the therapists to coherently integrate all the mobile phone's features into the clinical procedure. Moreover, the widespread introduction of the mobile phone requires considerable organisational changes in the way clinical treatments are delivered. This aspect first requires a modification of established factors in consultations and referral patterns. A further problem is the technological equipment; fortunately the quality of technology in this area is increasing while costs are decreasing.

At the time of writing, the limit of empirical research makes it impossible to objectively evaluate the benefits and the efficacy of the introduction of the mobile phone in clinical care. More evaluation of clinical outcomes, organisational effects, benefits to professionals' practice and patients, and quality assurance is required despite the encouraging findings reported here.

\section{Acknowledgements}

The present work was supported by the Italian MIUR FIRB programme (Project 'NeurotivManaged care basata su telepresenza immersiva virtuale per l'assessment e riabilitazione in 
neuro-psicologia e psicologia clinica' - RBNE01W8WH - and Project 'Realtà virtuale come strumento di valutazione e trattamento in psicologia clinica: aspetti tecnologici, ergonomici e clinici' - RBAU014JE5).

\section{Notes on contributors}

Alessandra Preziosa received her MS degree in Psychology from Catholic University of Milan. In 2008 she achieved the PhD studentship in Psycobiology at the University of Milano. Her main research interest concerns the study of new technologies, especially mobile phone technology, applied in social, communicative processes and in psychotherapy practice.

Alessandra Grassi received a Ms in Psychology from Catholic University of Milan. She is a $\mathrm{PhD}$ candidate and works in the Center of Study and Research in Communication Psychology in Catholic University. Her researches are focused on wellbeing improvement using new communication technology, in particular in the field of emotion regulation and work related stress disease.

Andrea Gaggioli received a MSc in Psychology from University of Bologna and a $\mathrm{PhD}$ from the Faculty of Medicine of the Public University of Milan. He is currently researcher at the Faculty of Psychology of the Catholic University of Milan and senior researcher at Istituto Auxologico Italiano (Milan, Italy). He focuses on Positive Technology, a field that studies how technology can be used to promote mental and physical wellbeing.

Giuseppe Riva, PhD, is Associate Professor of General Psychology and Communication Psychology at the Catholic University of Milan, Italy and Head Researcher of the the Applied Technology for Neuro-Psychology Laboratory - ATN-P Lab., Istituto Auxologico Italiano, Verbania Italy. He conducted several researches and published many papers about methods and assessment tools in psychology and about the use of Virtual Reality and Internet in medicine and in training.

\section{References}

Anthony, K. (2000). Counselling in cyberspace. Counselling Journal, 11(10), 625-627.

Anthony, K. (2005). Counselling problem gamblers online. Counselling \& Psychotherapy Journal, 16(6), 9-10.

Barrett, D.J., \& Feldman Barrett, L. (2001). An introduction to computerized experience sampling in psychology. Social Science Computer Review, 19(2), 175-185.

Bauer, S., Percevic, R., Okon, E., Meermann, R., \& Kordy, H. (2003). Use of text messaging in the aftercare of patients with bulimia nervosa. European Eating Disorders Review, 11(3), 279-290.

Baur, C. (2000). Limiting factors on the transformative powers of e-mail in patient-physician relationships: A critical analysis. Health Communication, 12(3), 239-259.

Braun, R.P., Vecchietti, J.L., Thomas, L., Prins, C., French, L.E., Gewirtzman, A.J., Saurat, J.H., \& Salomon, D. (2005). Telemedical wound care using a new generation of mobile telephones: A feasibility study. Archives of Dermatology, 141(2), 254-258.

Bloom, J.W. (1998). The ethical practice of webcounseling. British Journal of Guidance and Counselling, 26(1), 53-59.

Budman, S.H. (2000). Behavioral health care dot-com and beyond: Computer-mediated communications in mental health and substance abuse treatment. American Psychologist, 55(11), 1290-1300.

Castells, M. (1996). The information age: Economy, society, and culture. Oxford: Blackwell.

Chen, H. (2006). Digitization of the experience sampling method: Transformation, implementation, and assessment. Social Science Computer Review, 24(1), 106-118.

Cleland, J., Caldow, J., \& Ryan, D. (2007). A qualitative study of the attitudes of patients and staff to the use of mobile phone technology for recording and gathering asthma data. Journal of Telemedicine and Telecare, 13(2), 85-89.

Coburn, J., \& Manderino, M.A. (1986). Stress inoculation: An illustration of coping skills training. Rehabilitation Nursing, 11(1), 14-17. 
Collins, R.L., Kashdan, T.B., \& Gollnisch, G. (2003). The feasibility of using cellular phones to collect ecological momentary assessment data: Application to alcohol consumption. Experimental and Clinical Psychopharmacology, 11(1), 73-78.

Fortunati, L. (2002). Italy: Stereotypes, true and false. In J.E. Katz, M.A. Aakhus, \& A. Mark (Eds.), Perpetual contact. Mobile communication, private talk, public performance (pp. 4262). Cambridge: Cambridge University Press.

Gift, A. (1989). Visual analog scales: Measurement of subjective phenomenon. Nursing Research, 38(5), 286-288.

Green, A., Rafaeli, E., Bolger, N., Shrout, P.K., \& Reis, H.R. (2006). Paper or plastic? Data equivalence in paper and electronic diaries. Psychological Methods, 11(1), 87-105.

Griffiths, M. (2001). Online therapy: A cause for concern? The Psychologist, 14(4), 244-248.

Hains, A.A. (1992). A stress inoculation training program for adolescents in a high school setting: A multiple baseline approach. Journal of Adolescence, 15(2), 163-175.

Klein, B., \& Richards, C. (2001). A brief Internet-based treatment for panic disorder. Behavioural \& Cognitive Psychotherapy, 29(1), 536-542.

Jordan, S. (2005). www.drugcom.de: An Internet based information and counselling project for the prevention of addiction. Praxis der Kinderpsychologie und Kinderpsychiatrie, 54(9), 742-754.

Joyce, D., \& Weibelzahl, S. (2006). Text messaging as a means to lowering barriers to helpseeking in students with depression. Retrieved January 26, 2009, from http://www.easyhub.org/stephan/joyce-esociety06.pdf

Madara, E.J. (1990). Maximizing the potential for community self-help through clearinghouse approaches. Prevention in Human Services, 7(2), 109-138.

Maheu, M.M., \& Gordon, B.L. (2000). Counseling and therapy on the Internet. Professional Psychology: Research and Practice, 31(5), 484-489.

Manhal-Baugus, M. (2001). E-Therapy: Practical, ethical, and legal issues. CyberPsychology and Behavior, 4(5), 551-563.

Meichenbaum, D. (1996). Stress inoculation training for coping with stressors. The Clinical Psychologist, 49(4), 4-7.

Leong, K.C., Chen, W.S., Leong, K.W., Mastura, I., Mimi, O., Sheikl, M.A., Zailinawati, A.H., Ng, C.J., Phua, K.L., \& Teng, C.L. (2006). The use of text messaging to improve attendance in primary care: A randomized controlled trial. Family Practice, 23(6), 699-707.

Neville, R., Greene, A., McLeod, J., \& Tracy, A. (2002). Mobile phone text messaging can help young people manage asthma. British Medical Journal, 325(7364), 600.

Obermayer, J.L., Riley, W.T., Asif, O., \& Jean-Mary, J. (2004). College smoking-cessation using cell phone text messaging. Journal of American College Health, 53(2), 71-78.

Preziosa, A., Bassi, M., Villani, D., Gaggioli, A., \& Riva, G. (2006). Monitoring daily life using mobile phones: The experience sampling method. CyberPsychology and Behavior, 9(6), $711-712$.

Puro, J. (2002). Finland: A mobile culture. In J.E. Katz \& M.A. Aakhus (Eds.), Perpetual contact. Mobile communication, private talk, public performance (pp. 19-29). Cambridge: Cambridge University Press.

Robinson, S., Perkins, S., Bauer, S., Hammond, N., Treasure, J., \& Schmidt, U. (2006). Aftercare intervention through text messaging in the treatment of bulimia nervosaFeasibility pilot. International Journal of Eating Disorders, 39(8), 633-638.

Rodgers, A., Corbett, T., Bramley, D., Riddell, T., Wills, M., Lin, R.-B., \& Jones, M. (2005). Do u smoke after txt? Results of a randomised trial of smoking cessation using mobile phone text messaging. Tobacco Control, 14(4), 255-261.

Saunders, T., Driskell, J.E., Johnston, J.H., \& Salas, E. (1996). The effect of stress inoculation training on anxiety and performance. Journal of Occupational Health Psychology, 1(2), $170-186$.

Scogin, F., Bynum, J., Stevens, G., \& Calhoon, S. (1990). Efficacy of self-administered treatment program: Meta-analytic review. Professional Psychology: Research and Practice, 21(1), 42-47.

Spielberger, C.D., Gorsuch, R.L., Lushene, R., Vagg, P.R., \& Jacobs, G.A. (1983). Manual for the State-Trait Anxiety Inventory. Palo Alto, CA: Consulting Psychology Press.

Taylor, A.S., \& Harper, R. (2001). The gift of the gab? A design oriented sociology of young people's use of mobiles. Computer Supported Cooperative Work, 12(3), 267-296. 
Storr, A. (1990). The art of psychotherapy. New York: Routledge.

Watson, D., Clark, L.A., \& Tellegen, A. (1988). Development and validation of brief measures of positive and negative affect: The panas scales. Journal of Personality and Social Psychology, 54(6), 1063-1070.

World Telecommunication Development Report. (2002). 'Reinventing telecoms' \& trends in telecommunication reform 2002: 'Effective regulation'. Retrieved January 26, 2009, from http://www.itu.int/newsroom/wtdc2002/backgrounder.html 